\title{
Malnutrición en los tiempos del COVID-19
}

\section{Malnutrition on the COVID-19 time}

\author{
Sánchez PE $E^{1}$, Rosero $R J^{1}$, Stephens $I^{2}$
}

${ }^{1}$ Médico Internista y Endocrinólogo, Centro de Obesidad, Dismetabolismo y Deporte (COD2), Clínica Las Américas, Medellín, Colombia.

${ }^{2}$ Médica Internista y Endocrinóloga. Docente de Medicina, Universidad de los Andes. Servicio de Endocrinología, Fundación Santa fe de Bogotá, Colombia.

Autor de correspondencia: Pedro Enrique Sánchez Márquez Correo electrónico: pedro.sanchez@clincalasamericas.com.co

\section{Resumen}

La infección por el nuevo coronavirus SARS-CoV-2 ha puesto en jaque a la humanidad desde el punto de vista económico, político, cultural y sanitario. Su alta tasa de transmisibilidad y mortalidad nos ha hecho aprender y adaptarnos, de una forma rápida, a las nuevas condiciones que se presentan en el día a día. Con la experiencia actual y de pandemias previas, se han detectado múltiples factores asociados a peores desenlaces, entre los cuales se encuentra la hipertensión, la diabetes, la obesidad y la desnutrición. Esta no solo debe ser considerada como una baja masa corporal, sino también como la incapacidad para preservar una composición corporal saludable y una masa muscular esquelética adecuada. De igual forma, los niveles bajos de marcadores de estado nutricional son predictores de progresión a falla respiratoria y requerimiento de ventilación mecánica. Por tanto, en los pacientes infectados por SARS-CoV-2, la evaluación nutricional debe ser abordada como una prioridad, para evitar desenlaces deletéreos.

Palabras claves: desnutrición, COVID-19.

\section{Abstract}

Malnutrition should not only be considered as low body mass but as the inability to preserve a healthy body composition and adequate skeletal muscle mass. Malnutrition has been associated with greater severity and is a predictor of mortality. Similarly, low levels of nutritional markers are predictors of progression to respiratory failure and the requirement for mechanical ventilation. Therefore, in patients infected with SARS-CoV-2, nutritional evaluation should be approached as a priority to avoid deleterious outcomes.

Keywords: Malnutrition, COVID-19.

\section{Introducción}

La infección por el nuevo coronavirus SARS-CoV-2 ha tenido una tasa de diseminación en Colombia y en el mundo de proporciones inesperadas, con más de 230.000 muertes a la fecha a nivel global ${ }^{(1)}$. Se ha documentado que la mayoría de las complicaciones graves y muertes luego de la infección se presenta en adultos mayores y personas con polimorbilidades, como enfermedad cardiovascular, hepática, renal y cáncer ${ }^{(2-4)}$, los cuales se caracterizan por un alto riesgo nutricional.

La desnutrición no solo debe ser considerada como una baja masa corporal, sino también como la incapacidad para preservar una composición corporal saludable y una masa muscular esquelética adecuada. El estado nutricional debe ser evaluado en todos los pacientes infectados con SARS-CoV-2 al momento de la admisión ${ }^{(5,6)}$. Para la identificación del riesgo, se puede utilizar herramientas de tamizaje, como los criterios MUST ${ }^{(7)}$, o en pacientes hospitalizados el NRS-2002 ${ }^{(8)}$. En pacientes hospitalizados en la unidad de cuidados intensivos (UCI) se han utilizado otras herramientas, como el NUTRIC Score ${ }^{(9)}$ y el GLIM ${ }^{(10)}$. En este último, primero se identifica el estado de riesgo y luego se evalúa el diagnóstico y el grado de gravedad de la malnutrición, para lo cual se necesita al menos un criterio fenotípico y un criterio etiológico. Según la experiencia en China, se sugirió que el estado nutricional para cada paciente infectado debe ser evaluado antes de la administración de tratamientos generales ${ }^{(11)}$.

Para evaluar las necesidades nutricionales también se pueden utilizar distintos métodos, incluidas ecuaciones predictivas y calorimetría indirecta. El requerimiento proteico es de al menos $1 \mathrm{~g} / \mathrm{kg} / \mathrm{d}$ en adultos mayores, siendo mayor en personas con comorbilidades para prevenir la pérdida de peso y reducir el riesgo de complicaciones, reingresos y muerte. El requerimiento de grasas y carbohidratos se ajusta a los requerimientos energéticos, considerando una relación de 30:70 en sujetos sin insuficiencia respiratoria y de 50:50 en pacientes ventilados ${ }^{(12)}$.

Análisis retrospectivos de las pandemias previas han mostrado que la gravedad del cuadro depende de los factores del virus y del huésped. Entre los factores del huésped se encuen- 
tra el estado nutricional, la respuesta inmunitaria celular y humoral y la genética ${ }^{(11)}$. En infecciones virales y bacterianas, la malnutrición incluso se ha asociado con mayor gravedad y mortalidad en la población. En análisis multivariados de otros tipos de infecciones virales, como la influenza, la desnutrición se ha visto como un predictor de mortalidad (OR: 25,0) ${ }^{(13)}$. Bajos niveles de prealbúmina han demostrado ser un factor predictor de progresión a falla respiratoria y requerimiento de ventilación mecánica ${ }^{(14)}$, por lo cual, en los pacientes infectados por SARS-CoV-2, la nutrición debe ser manejada de forma prioritaria, considerando que la respuesta inmunitaria está debilitada por una reducción en la ingesta ${ }^{(15)}$, aunado a una disminución del movimiento, los cambios catabólicos y el estado proinflamatorio, que pueden contribuir a la presencia de pobres desenlaces en este tipo de pacientes ${ }^{(16)}$.

La mayoría de los pacientes infectados por SARS-CoV-2 presentan inflamación grave y anorexia, que, asociadas a manifestaciones gastrointestinales tales como náuseas, vómito y diarrea, alteran la ingesta y la absorción de la comida ${ }^{(17)}$, por lo que una buena nutrición es una ventaja para las personas en riesgo. Los sujetos con malnutrición deben buscar optimizar su estado nutricional, idealmente con asesoría de personal especializado; preservar el estado nutricional y prevenir o tratar la malnutrición tiene el potencial de reducir las complicaciones y los desenlaces negativos en pacientes que pueden presentar infección por SARS-CoV-2 en el futuro.

La prevalencia de malnutrición en adultos mayores con CoV-2 es elevada. En un estudio realizado en el Hospital Tongji, de Wuhan ${ }^{(18)}$, en pacientes mayores de 65 años que fueron sometidos a una evaluación de su estado nutricional por medio del cuestionario MNA (mininutritional assesment) ${ }^{(19)}$; en los cuales se consideró un valor menor de 17 puntos como malnutrición, entre 17 y 23,5 puntos como riesgo de malnutrición y entre 24 y 30 puntos como sin malnutrición; en 182 casos evaluados se encontró que el 52,7\% ( $\mathrm{n}=96)$ presentaba malnutrición y el 27,5\% ( $n=50)$ estaba en riesgo de presentarla. No se encontraron diferencias en cuanto a la edad, el género y las comorbilidades entre los tres grupos, en términos de antropometría. El único valor que se correlacionó fue el nivel bajo de circunferencia de la pantorrilla. El análisis multivariado que combinó diabetes (OR: 2,12; IC: 1,92-3,21), baja circunferencia de la pantorrilla (OR: 2,98; IC 95\%: 2,29-3,53) y bajo nivel de albúmina (OR: 2,98; IC 95\%: 2,43-5,19) mostró que estos tres eran factores de riesgo independientes para malnutrición.

\section{Intervención nutricional}

A pesar de la escasa evidencia en el abordaje nutricional en el paciente con infección por SARS-CoV-2, es fundamental evaluar el estado nutricional desde el inicio de la hospitalización o del diagnóstico, sobre todo en los pacientes mayores de 65 años y con comorbilidades tales como diabetes, insuficiencia cardíaca y EPOC, que se asocian a sarcopenia ${ }^{(20)}$, y/o pacientes con bajo diámetro de la circunferencia de la pantorrilla. También deben implementarse tempranamente todas las medidas que busquen recuperar el adecuado estado nutricional del paciente, puesto que la malnutrición afectará a los pacientes con esta enfermedad.

Una vez sean detectados los estados de malnutrición, pueden utilizarse suplementos nutricionales orales (SNO) ${ }^{(6)}$, que deberán individualizarse según las necesidades del paciente. Incluso los pacientes de bajo riesgo con SARS-CoV-2 deben mantener ingestas diarias de proteína, de 1,5 g/d, y calóricas, de 25-30 kcal/d (6), además del consumo de vitaminas, que ha mostrado un efecto antiinflamatorio y antioxidante ${ }^{(5)}$, vitaminas liposolubles (A, D, E y C), entre otras, junto con zinc, hierro, cobre y selenio. En los estudios se ha observado cómo el zinc y la piritiona, en bajas concentraciones, pueden inhibir la replicación del coronavirus del SARS, como coadyuvantes en la respuesta inmunitaria ${ }^{(21)}$. Sin embargo, su deficiencia se relaciona con desenlaces desfavorables en infecciones virales ${ }^{(22)}$, ya sea por una inadecuada respuesta inmunitaria o por la afectación directa del patógeno.

La hiporexia, como una característica asociada, lleva al bajo consumo proteico, por lo que algunas revisiones han sugerido la suplementación de proteínas de suero orales ( $20 \mathrm{~g} / \mathrm{d})$, multivitaminas intravenosas, multiminerales y soluciones traza desde la admisión ${ }^{(23)}$. Las proteínas de suero han sido recomendadas, particularmente por el beneficio anabólico y antioxidante, combinado con la alta digestibilidad ${ }^{(24)}$ y las propiedades inmunomoduladoras ${ }^{(25)}$ con potencial actividad antiviral ${ }^{(26)}$. En pacientes con riesgo nutricional establecido, el inicio de los SNO está dirigido a garantizar un aporte de 35-55 g/d de proteínas con 600-900 $\mathrm{kcal} / \mathrm{d}$, adicionados con las comidas ${ }^{(23)}$.

Los pacientes críticos, con complicaciones respiratorias agudas que requieren estancia prolongada en la UCI, se asocian a mayor morbilidad y mortalidad ${ }^{(3,17,27)}$ y a deficiencia nutricional, con pérdida de la masa y función muscular esquelética, lo cual puede llevar a pobre calidad de vida, discapacidad y morbilidades al egreso de la UCI ${ }^{(28)}$. Las recomendaciones nutricionales de la Asociación Colombiana de Nutrición Clínica para pacientes hospitalizados con infección por SARS-CoV-2 sugieren considerar a todo paciente hospitalizado en UCI por más de 48 horas en riesgo nutricional y realizar una valoración general del estado nutricional que incluya anamnesis, valoración clínica, antropometría y marcadores bioquímicos ${ }^{(21)}$.

En caso de que la suplementación oral no sea tolerada y que la vía enteral genere compromiso en la efectividad de las medidas de ventilación por la presencia de sonda nasogástrica o la dilatación gástrica, que puede afectar la función diafragmática y la efectividad de la ventilación no invasiva ${ }^{(29)}$, se plantea la opción de nutrición parenteral ${ }^{(30,31)}$. Además, en estudios comparativos realizados en pacientes críticos, se observó que la nutrición enteral ha mostrado una mayor incidencia de eme- 
sis, diarrea y complicaciones gastrointestinales, sin diferencias en la mortalidad ${ }^{(32)}$. En el contexto de los pacientes con SARS$\mathrm{CoV}-2$, en quienes se ha reportado síntomas gastrointestinales, se ha observado una progresión más rápida y peores desenlaces ${ }^{(33)}$, lo cual altera la tolerancia de la vía enteral.

En los pacientes críticos puede implementarse nutrición isocalórica luego de la fase aguda de la enfermedad. Si la calorimetría no está disponible, la medición del consumo de oxígeno $\left(\mathrm{VO}_{2}\right)$ del catéter de la arteria pulmonar o la producción de dióxido de carbono $\left(\mathrm{VCO}_{2}\right)$ derivado del ventilador proporcionan una evaluación del gasto energético mejor que las fórmulas predictivas. Una nutrición hipocalórica que no exceda el 70\% del gasto energético debe darse en la fase temprana aguda de la enfermedad, con un incremento al 80\%-100\% luego del día 3. Los requerimientos proteicos en los días críticos deben ser de $1,3 \mathrm{~g} / \mathrm{kg} / \mathrm{d}$ ajustados al peso ideal; este objetivo ha mostrado una mejoría en la sobrevida de los pacientes frágiles ${ }^{(34)}$.

\section{Conclusión}

La malnutrición es un factor de riesgo independiente, que se asocia a mayor gravedad de la enfermedad y mortalidad, no dependiente del grupo etario. Por esto, la implementación pronta y apropiada de los cuidados nutricionales en los pacientes con COVID-19 debe ser una medida temprana y prioritaria para evitar los desenlaces deletéreos.

\section{Conflicto de interés}

Ninguno.

\section{Financiación}

Ninguna.

\section{Agradecimientos}

Queremos agradecer a la Asociación Colombiana de Endocrinología por el compromiso y apoyo continuo en la educación y participación científica en temas de alta relevancia.

\section{Referencias}

1. Jhons Hopkins University \& Medicine. COVID-19 Dashboard by the Center for Systems Science and Engineering (CSSE) at Johns Hopkins [Internet]. Disponible en: https://coronavirus.jhu.edu/map.html

2. Guan WJ, Ni ZY, Hu Y, Liang WH, Ou CQ, He JX, et al. Clinical Characteristics of Coronavirus Disease 2019 in China. N Engl J Med. 2020;382(18):170820. doi: 10.1056/NEJMoa2002032.

3. Huang C, Wang Y, Li X, Ren L, Zhao J, Hu Y, et al. Clinical features of patients infected with 2019 novel coronavirus in Wuhan, China. Lancet. 2020;395(10223):497-506. doi: 10.1016/S0140-6736(20)30183-5.

4. Wang T, Du Z, Zhu F, Cao Z, An Y, Gao Y, et al. Comorbidities and multi-organ injuries in the treatment of COVID-19. Lancet. 2020;395(10228):e52. doi: 10.1016/S0140-6736(20)30558-4.

5. Zhang L, Liu Y. Potential interventions for novel coronavirus in China: A systematic review. J Med Virol. 2020;92(5):479-90. doi: 10.1002/ jmv.25707.

6. Jin YH, Cai L, Cheng ZS, Cheng H, Deng T, Fan YP, et al. A rapid advice guideline for the diagnosis and treatment of 2019 novel coronavirus (2019$\mathrm{nCoV}$ ) infected pneumonia (standard version). Mil Med Res. 2020;7(4). doi: 10.1186/s40779-020-0233-6.

7. Malnutrition Advisory Group (MAG). Malnutrition Universal Screening Tool (MUST) - Instrumento universal para el cribado de la malnutrición [Internet]. Disponible en: https://www.bapen.org.uk/images/pdfs/ must/spanish/must-toolkit.pdf.

8. Kondrup J. Nutrition Risk Screening 2002 (NRS-2002) [Internet]. Disponible en: https://www.mdcalc.com/nutrition-risk-screening2002-nrs-2002.

9. Volkert D, Beck AM, Cederholm T, Cruz-Jentoft A, Goisser S, Hooper L, et al. ESPEN guideline on clinical nutrition and hydration in geriatrics. Clin Nutr. 2019;38(1):10-47. doi: 10.1016/j.clnu.2018.05.024.

10. Cederholm T, Jensen GL, Correia MITD, Gonzalez MC, Fukushima R, Higashiguchi T, et al. GLIM criteria for the diagnosis of malnutrition - A consensus report from the Global Clinical Nutrition Community. Clin Nutr. 2019;38(1):1-9. doi: 10.1016/j.clnu.2018.08.002.

11. Short KR, Kedzierska K, van de Sandt CE. Back to the Future: Lessons Learned From the 1918 Influenza Pandemic. Front Cell Infect Microbiol. 2018;8(343). doi: 10.3389/fcimb.2018.00343.
12. Gomes F, Schuetz P, Bounoure L, Austin P, Ballesteros-Pomar M, Cederholm T, et al. ESPEN guidelines on nutritional support for polymorbid internal medicine patients. Clin Nutr. 2018;37(1):336-53. doi: 10.1016/j. clnu.2017.06.025.

13. Reyes L, Arvelo W, Estevez A, Gray J, Moir JC, Gordillo B, et al. Population-based surveillance for 2009 pandemic influenza A (H1N1) virus in Guatemala, 2009. Influenza Other Respi Viruses. 2010;4(3):129-40. doi: 10.1111/j.1750-2659.2010.00138.x.

14. Wu C, Chen X, Cai Y, Xia J, Zhou X, Xu S, et al. Risk Factors Associated with Acute Respiratory Distress Syndrome and Death in Patients with Coronavirus Disease 2019 Pneumonia in Wuhan, China. JAMA Intern Med. 2020;e200994. doi: 10.1001/jamainternmed.2020.0994.

15. Yaqoob P. Ageing alters the impact of nutrition on immune function. Proc Nutr Soc. 2017;76(3):347-51. doi: 10.1017/S0029665116000781.

16. Lomax AR, Calder PC. Prebiotics, immune function, infection and inflammation: A review of the evidence. Br J Nutr. 2009;101(5):633-58. doi: $10.1017 /$ S0007114508055608.

17. Chen N, Zhou M, Dong X, Qu J, Gong F, Han Y, et al. Epidemiological and clinical characteristics of 99 cases of 2019 novel coronavirus pneumonia in Wuhan, China: a descriptive study. Lancet. 2020;395(10223):507-13. doi: 10.1016/S0140-6736(20)30211-7.

18. Li T, Zhang Y, Gong C, Wang J, Liu B, Shi L, et al. Prevalence of malnutrition and analysis of related factors in elderly patients with COVID-19 in Wuhan, China. Eur J Clin Nutr. 2020;1-5. doi: 10.1038/s41430-020-0642-3.

19. Guigoz Y, Lauque S, Vellas BJ. Identifying the elderly at risk for malnutrition the mini nutritional assessment. Clin Geriatr Med. 2002;18(4):73757. doi: 10.1016/s0749-0690(02)00059-9.

20. Chung HY, Cesari M, Anton S, Marzetti E, Giovannini S, Seo AY, et al. Molecular inflammation: Underpinnings of aging and age-related diseases. Ageing Res Rev. 2009;8(1):18-30. doi: 10.1016/j.arr.2008.07.002.

21. Bermúdez C, Pereira F, Trejos-Gallego D, Pérez A, Puentes M, María López L, et al. Recomendaciones nutricionales de la Asociación Colombiana de Nutrición Clínica para pacientes hospitalizados con infección por SARSCoV-2. Nutr Clin Metab. 2020;3(1):74-85. doi: https://doi.org/10.35454/ rncm.v3n1.066. 
22. Semba RD, Tang AM. Micronutrients and the pathogenesis of human immunodeficiency virus infection. Br J Nutr. 1999;81(3):181-9. doi: $10.1017 /$ s0007114599000379.

23. Caccialanza R, Laviano A, Lobascio F, Montagna E, Bruno R, Ludovisi S, et al. Early nutritional supplementation in non-critically ill patients hospitalized for the 2019 novel coronavirus disease (COVID-19): Rationale and feasibility of a shared pragmatic protocol. Nutrition. 2020;74:110835. doi: 10.1016/j.nut.2020.110835.

24. McPherson RA, Hardy G. Clinical and nutritional benefits of cysteine-enriched protein supplements. Curr Opin Clin Nutr Metab Care. 2011;14(6):562-8. doi: 10.1097/MC0.0b013e32834c1780.

25. Cross ML, Gill HS. Immunomodulatory properties of milk. Br J Nutr 2000;84(Suppl 1):S81-9. doi: 10.1017/s0007114500002294.

26. Ng TB, Cheung RCF, Wong JH, Wang Y, Ip DTM, Wan DCC, et al. Antiviral activities of whey proteins. Appl Microbiol Biotechnol. 2015;99(17):69977008. doi: $10.1007 /$ s00253-015-6818-4.

27. Zhu N, Zhang D, Wang W, Li X, Yang B, Song J, et al. A novel coronavirus from patients with pneumonia in China, 2019. N Engl J Med. 2020;382(8):72733. doi: $10.1056 /$ NEJMoa2001017.

28. Singer P, Blaser AR, Berger MM, Alhazzani W, Calder PC, Casaer MP, et al. ESPEN guideline on clinical nutrition in the intensive care unit. Clin Nutr. 2019;38(1):48-79. doi: 10.1016/j.clnu.2018.08.037.
29. Leder SB, Siner JM, Bizzarro MJ, McGinley BM, Lefton-Greif MA. Oral Alimentation in Neonatal and Adult Populations Requiring High-Flow Oxygen via Nasal Cannula. Dysphagia. 2016;31(2):154-9. doi: 10.1007/ s00455-015-9669-3.

30. Singer P, Rattanachaiwong S. To eat or to breathe? The answer is both Nutritional management during noninvasive ventilation. Crit Care 2018;22(1):7. doi: 10.1186/s13054-018-1947-7.

31. Kogo M, Nagata K, Morimoto T, Ito J, Sato Y, Teraoka S, et al. Enteral nutrition is a risk factor for airway complications in subjects undergoing noninvasive ventilation for acute respiratory failure. Respir Care. 2017;62(4):459-67. doi: 10.4187/respcare.05003.

32. Doig GS, Simpson F, Sweetman EA, Finfer SR, Cooper J, Heighes PT, et al. Early parenteral nutrition in critically ill patients with short-term relative contraindications to early enteral nutrition: A randomized controlled trial. JAMA. 2013:309(20):2130-8. doi: 10.1001/jama.2013.5124.

33. Pan L, Mu M, Yang P, Sun Y, Wang R, Yan J, et al. Clinical Characteristics of COVID-19 Patients With Digestive Symptoms in Hubei, China: A Descriptive, Cross-Sectional, Multicenter Study. Am J Gastroenterol. 2020;115(5):766-73. doi: 10.14309/ajg.0000000000000620.

34. Barazzoni R, Bischoff SC, Breda J, Wickramasinghe K, Krznaric Z, Nitzan D et al. ESPEN expert statements and practical guidance for nutritional management of individuals with SARS-CoV-2 infection. Clin Nutr. 2020;S0261 5614(20)30140-0. doi: 10.1016/j.clnu.2020.03.022. 